\title{
WORKPIECE TEMPERATURE VARIATIONS DURING FLAT PERIPHERAL GRINDING
}

\author{
Vitalii A. SMIRNOV, Aleksandr V. REPKO \\ Votkinsk Branch of Kalashnikov Izhevsk State Technical University
}

\begin{abstract}
:
The paper presents the results of researches of temperature variations during flat peripheral grinding. It is shown that the temperature variations of the workpiece can reach $25 . . .30 \%$ of the average values, which can lead to some thermal defects. A nonlinear two-dimensional thermophysical grinding model is suggested. It takes into account local changes in the cutting conditions: the fluctuation of the cut layer and the cutting force, the thermal impact of the cutting grains, and the presence of surface cavities in the intermittent wheel. For the numerical solution of the problem, the method of finite differences is adapted. Researches of the method stability and convergence are made, taking into account the specific nature of the problem. A high accuracy of the approximation of the boundary conditions and the nonlinear heat equation is provided. An experimental verification of the proposed thermophysical model was carried out with the use of installation for simultaneous measurement of the grinding force and temperature. It is shown that the discrepancy between the theoretical and experimental values of the grinding temperature does not exceed $5 \%$. The proposed thermophysical model makes it possible to predict with high accuracy the temperature variations during grinding by the wheel periphery.
\end{abstract}

Key words: cutting force, cutting grains, flat grinding, temperature variations

\section{INTRODUCTION}

Thermal processes occupy a central place in the research of grinding. This particularly applies to grinding hard -to-machine materials: titanium alloys, high-strength steels, tool steels and other materials. Understanding thermal processes allows you to choose the optimum grinding wheel parameters and grinding modes while maintaining the quality of the machining surfaces.

Local overheating of the surface layer can lead to:

- the occurrence of thermal grinding defects (burns, microcracks, thermoplastic deformation) $[5,7,11]$;

- an unfavorable change in the properties of the surface layer (softening, local tempering, chemical interaction with the environment, stretching residual stresses) $[1,5,6,7,12]$;

- the activation of the physicochemical process of wear of the grinding wheel grains $[7,12]$.

Usually the problem of thermal defects is solved by a decrease in the productivity of the grinding process, which means an increase in the time and cost of processing.

\section{METHODOLOGY OF RESEARCH}

The known methods for calculating the grinding temperature $[3,5,6]$ do not take into account a number of factors.

1. Contact conditions of the wheel and the workpiece vary in time due to small amplitude vibrations of the technological system elements and uneven wear of the grinding wheel [6]. Variable contact conditions can lead to local overheating of the surface layer.
2. The workpiece undergoes a local high-frequency thermal impact when cutting with a wheel grain, which determines the instantaneous grinding temperature. This is clearly confirmed by the results of the researches [4, 13].

3. When grinding, there are large local temperature gradients along the depth of the workpiece (up to $2000^{\circ} \mathrm{C} / \mathrm{mm}$ ) and in time (up to $105^{\circ} \mathrm{C} / \mathrm{s}$ ). This makes special demands on the numerical method for calculating the thermophysical grinding model.

These features of the grinding process are confirmed experimentally. Measurements by the method of a semiartificial thermocouple show that the grinding temperature has variations in $25 . . .30 \%$ during the passage of the grinding wheel along the measuring electrode (see Fig. 5). Temperature variations increase with increasing wear of grinding wheel grains.

We have obtained a mathematical model for determining the dynamic coefficient, which characterizes the influence of vibrations in the technological system on the value of the cut-off layer when peripheral grinding $[9,14]$ :

$$
k_{a}=1+\frac{2 \pi v A}{V_{f}} \cdot \sqrt{\frac{R}{2 a_{p}}}
$$

where:

$v, A$ is the frequency and amplitude of vibrations in the radial direction,

$a_{p}$ is the cutting depth, $R$ is the radius of the wheel, $V_{f}$ is the feed rate. 
Analysis of formula (1) shows that even small amplitudes of fluctuations A lead to significant fluctuations of the cut layer (and therefore the grinding temperature). For example, for $A=1 \mu \mathrm{m}, v=50 \mathrm{~Hz}, \mathrm{R}=100 \mathrm{~mm}, \mathrm{a}_{\mathrm{p}}=0.01$ $\mathrm{mm}, \mathrm{V}_{\mathrm{f}}=3 \mathrm{~m} / \mathrm{min}, \mathrm{k}_{\mathrm{a}}=1.44$. This means that the average thickness of the cut layer can fluctuate within $\pm 44 \%$.

Let us now consider the thermophysical model of flat peripheral grinding, as well as the numerical realization of the model.

\section{Thermophysical grinding model}

The mathematical model is based on the nonlinear nonstationary two-dimensional heat equation:

$$
c(\Theta) \rho \frac{\partial \Theta(y, z, \tau)}{\partial \tau}=\operatorname{div}(\lambda(\Theta) \nabla \Theta(y, z, \tau))
$$

where:

$\tau$ is time,

$y$ coordinate in depth of the workpiece,

$z$ coordinate along the workpiece,

$\Theta(y, z, \tau)$ is the temperature at the point with the coordinates $y$,

$z$ at time $\tau$,

$\lambda(\Theta), c(\Theta)$ coefficient of thermal conductivity and specific heat of the workpiece material, depending on temperature $\Theta$, $\rho$ is the density of the workpiece material.

A nonlinear problem is considered, since when heating to several hundred degrees the thermophysical properties of the workpiece material vary significantly. face:

The boundary conditions are set on the processed sur-

- non-stationary mobile heat flow in the contact area of the grinding wheel with the workpiece:

$$
\lambda(\Theta) \frac{\partial \Theta}{\partial y}=q(z, \tau)
$$

- cooling by liquid flow outside the contact zone:

$$
\lambda(\Theta) \frac{\partial \Theta}{\partial y}=\alpha\left(\Theta-\Theta_{f}\right)
$$

where:

$\alpha$ is the coefficient of heat transfer of the coolant,

$\Theta_{f}$ is the temperature of the coolant.

With peripheral grinding, the contact area of the wheel and the workpiece is approximately a rectangle with sides $B$ and $L_{c}$ (Fig. 1). The heat flow in the cutting zone is proposed to be divided into 2 parts: a distributed flow of density $q_{0}$ and an impulse flow of density $q_{1}$. According to our observations, this method of partitioning leads to the most exact agreement with the experimental data. The distributed part $q_{0}$ gives the overall thermal background of the workpiece, and the impulse part $q_{1}$ simulates local temperature surges from the operation of the single wheel grains. The value of $q_{0}$ can vary with time and moves along the $z$ axis with the feed rate $V_{f}$. The impulse part $q_{1}$ moves relative to $q_{0}$ with the peripheral velocity $V$. The quantity $q_{1}$ is considered as random, since the grains in the wheel are located chaotically.

In Fig. 1:

$B, L_{c}-$ width and length of the contact of the grinding wheel and workpiece,

$d_{w}$ is the average diameter of the contact surface of the wheel grains.

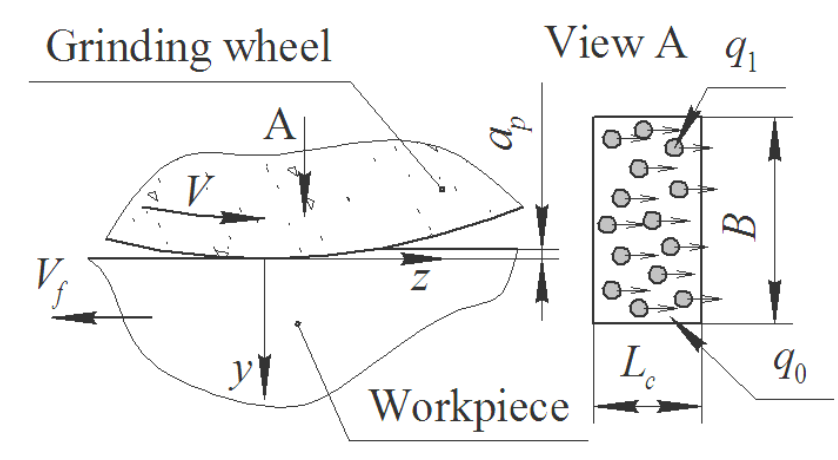

Fig. 1 To the definition of boundary conditions in the contact zone

The values of $q_{0}$ and $q_{1}$ are calculated from the following conditions:

1. The values $q_{0}$ and $q_{1}$ form the total power of the heat flow:

$$
q B L_{c}=q_{0} B L_{c}+\overline{q_{1}} \cdot \frac{n_{c u t} L_{\mathrm{c}}}{2 \pi R} \cdot \frac{\pi d_{w}^{2}}{4}
$$

where:

$\mathrm{n}_{\text {cut }}$ is the number of cutting grains on the wheel surface,

$\bar{q}_{1}$ is the average value of $q_{1}$.

2. The coefficient of heat flow unbalance is set - the ratio between the largest value of $q_{1 \max }$ and the mean value of $q_{0}$.

The value of $k_{u}$ is determined from the results of processing the experimental data of the grinding temperature. In the calculations it is assumed that $\mathrm{q}_{1}$ is random and distributed in the interval $\left[0 ; q_{1 \text { max }}\right.$. Assume that $\bar{q}_{1}=0.5$ $q_{1 \max }=0.5 q_{0} \cdot k_{u}$. When performing numerical simulation, $q_{1}$ is generated as a random value for each cutting grain.

The total density of the heat flow is determined by the formula:

$$
q(\tau)=i(\tau) \cdot \alpha_{w} \frac{F_{t}(\tau) V}{B L_{\mathrm{c}}}
$$

where:

$F_{t}$ is the tangential force (varies with time due to small vibrations in the technological system),

$$
F_{t}(\tau)=F_{t 0}+\sum_{i} A_{F t i} \cdot \sin \left(2 \pi v_{i}+\psi_{i}\right)
$$

$\alpha_{w}$ is the fraction of heat leaving the cutting zone into the workpiece,

$\mathrm{V}$ is the peripheral speed of the grinding wheel,

$i(\tau)$ is a function for intermittent grinding wheels $(i(\tau)=0)$ when passing a wheel groove,

$i(\tau)=1$ when passing the cutting surface).

As a result, from condition (5) we obtain:

$$
q_{0}=\frac{16 \alpha_{w} R V \cdot i(\tau) \cdot F_{t}(\tau)}{L_{\mathrm{c}}\left(16 R B+k_{u} n_{c u t} d_{w}^{2}\right)}
$$

Adaptive numerical method for solving the thermophysical problem

For numerical realization of the thermophysical model, it is proposed to use the adaptive numerical method of finite differences. The method has been adapted in two ways: increasing the accuracy of approximation of the boundary conditions ( 3 ) and (4), increasing the accuracy of solving the nonlinear heat equation (1) by minimizing the total error of convergence and instability of the solution. 
A large temperature gradient near the workpiece surface requires the use of a small grid pitch along the $y$ coordinate and a finite-difference approximation of high accuracy for the derivative $d \Theta / d y$. It is proposed to use the third-order accuracy approximation, obtained by the method of undetermined coefficients:

$$
\frac{\partial \Theta}{\partial y} \approx \frac{1}{6 \Delta y}\left(-11 \Theta_{i, 0}^{k+1}+18 \Theta_{i, 1}^{k+1}-9 \Theta_{i, 2}^{k+1}+2 \Theta_{i, 3}^{k+1}\right)+O\left(\Delta y^{3}\right)
$$

where:

$\Theta_{i, j}^{k}$ is the temperature at the cell $(i, j, k) ; i, j$,

$k$ indices characterizing the position of the calculated element along the $z, y, \tau$ axes,

$\Delta y$ is the grid pitch along the $y$-coordinate. According to our calculations, approximating of order above the third does not give a significant increase in accuracy.

The choice of the finite-difference scheme for the heat equation was based on the following assumptions.

1. It is necessary to take a small grid pitch along the depth of the workpiece $\Delta y$ and in time $\Delta \tau$, which is connected with ensuring the convergence of the solution for large temperature gradients. In practice, this leads to the need to calculate the temperature in the number of cells of the order of $10^{8} \ldots 10^{9}$.

2. The use of implicit schemes for finite-difference approximation is impractical, since this will require solving tens of thousands of linear equations systems consisting of tens of thousands of equations. In this case, the systems of equations are not three-diagonal, which makes it impossible to use the productive sweep method. Therefore, it is decided to use an explicit scheme for calculating the temperature at the cells.

3. The question of the method sensitivity to the choice of grid pitches requires solution. It is necessary to choose a finite-difference approximation for which the total error of instability and approximation is minimal when using the required grid pitches.

It is suggested to use the following explicit scheme for the finite-difference approximation of the heat equation:

$$
\begin{gathered}
c_{i, j} \rho \cdot \frac{\Theta_{i, j}^{k+1}-\Theta_{i, j}^{k}}{\Delta \tau}= \\
=\lambda_{i, j} \cdot\left(\frac{\Theta_{i, j+1}^{k}-2\left(\sigma \Theta_{i, j}^{k+1}+(1-\sigma) \Theta_{i, j}^{k}\right)+\Theta_{i, j-1}^{k}}{\Delta y^{2}}+\right. \\
\left.+\frac{\Theta_{i+1, j}^{k}-2\left(\sigma \Theta_{i, j}^{k+1}+(1-\sigma) \Theta_{i, j}^{k}\right)+\Theta_{i-1, j}^{k}}{\Delta z^{2}}\right)
\end{gathered}
$$

where:

$\sigma$ is a variable parameter $(\sigma=0 \ldots 1)$.

Using the method of harmonic analysis [2], a sufficient condition for the scheme stability is obtained:

$$
\Delta \tau \leq \frac{1}{(1-\sigma) \cdot\left(\frac{1}{\Delta y^{2}}+\frac{1}{\Delta z^{2}}\right) \cdot \frac{2 \lambda}{c \rho}}
$$

It is also established that scheme (9) has the following accuracy order [2]:

$$
O(\Delta \tau)+O\left(\Delta y^{2}\right)+O\left(\Delta z^{2}\right)+2 \sigma \cdot\left(O\left(\frac{\Delta \tau}{\Delta y^{2}}\right)+O\left(\frac{\Delta \tau}{\Delta z^{2}}\right)\right)
$$

As the parameter $\sigma$ increases, the approximation error increases due to the last term of formula (11). It can be expected that as the parameter $\sigma$ increases, the instability error of the solution decreases, since the stability condition (10) becomes less stringent. The last term of formula (11) shows that the scheme (9) is conditionally convergent: as the grid pitches $\Delta y$ and $\Delta z$ decrease, it is necessary to reduce the grid pitch $\Delta \tau$. A similar conclusion can be reached by analyzing the stability condition (10). By changing the parameter $\sigma$, one can optimize the accuracy of the numerical solution.

The results of calculations showed that for $\sigma=0.5$ the obtained solution depends weakly on the chosen grid pitches. This indicates a small error in the numerical method. It is established that the error of the method depends on the thermophysical properties of the workpiece material. For example, for titanium alloys, the error was less than $0.25 \%$, for stainless steels - less than $0.7 \%$, for tool and high-speed steels - less than $0.9 \%$, for structural steel - less than $1.3 \%$. With increasing of the workpiece thermal conductivity, the error of the numerical method slightly increases, but usually does not exceed $1 \%$.

The proposed mathematical model and numerical method are implemented in the special software that allows you to automate calculations and visualize the result. The software is based on the C\# platform. To increase performance, most of the calculations are performed in an unmanaged $\mathrm{C}++$ dll-library.

\section{Experimental researches methods}

The next step is to check the adequacy of the developed thermophysical model. In Fig. 2 there is a schematic diagram of an experimental installation for simultaneous measurement of cutting force and grinding temperature. To measure the temperature, a semi-artificial thermocouple 2 is used, where one of the electrodes is made of the test material. Such method makes it possible to exclude the inertia characteristic of an artificial thermocouple. To measure the cutting force, a tensometric sensor 4 is used. The signals from the sensors are fed to two analog-to-digital converters LTR27 and LTR212, mounted in a single frame and under the control of the LTR U-8-1 rack system. The received digital signals are processed on a personal computer using the special software for processing data ACTest.

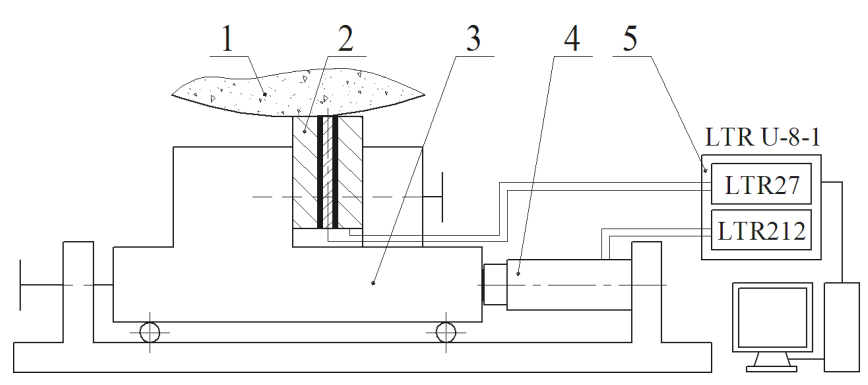

Fig. 2 Diagram of the experimental installation for measuring the cutting force and grinding temperature

1 - grinding wheel, 2 - semi-artificial thermocouple, 3 - movable part of dynamometer, 4 - tensometric sensor, 5 - analog-todigital converters.

\section{RESULTS OF RESEARCH}

\section{Results of mathematical modeling}

Consider the results of modeling, which can be obtained by developed thermophysical model and software. 
As an example, consider the grinding process of a titanium alloy Ti-6Al-4V with a silicon carbide grinding wheel. We take the following initial data for the calculation: $R=100$ $\mathrm{mm} ; \mathrm{B}=16 \mathrm{~mm} ; \mathrm{V}=35 \mathrm{~m} / \mathrm{s} ; \mathrm{V}_{\mathrm{f}}=3 \mathrm{~m} / \mathrm{min}$; grit $-\mathrm{F} 40 ; \mathrm{F}_{\mathrm{to}}=$ $20 \mathrm{~N}$ - average cutting force; $\alpha=3000 \mathrm{~W} / \mathrm{m}^{2 \circ} \mathrm{C}$.

Fig. 3 shows the theoretical graph for the grinding temperature variations: a - basic calculation with a constant heat flow, $b$ - case "a" with the addition of vibrations and the addition of cutting with single grains, $c$ - case " $b$ " for a grinding wheel with intermittent surface.

Results of the experimental verification

Experimental researches were carried out in the following areas.

a. Comparison of cutting force fluctuations and temperature variations.

The obtained cutting force signals and the workpiece temperature are laid out into the spectrum, so the dominant frequencies and amplitudes of the fluctuations are determined. An example of a research for grinding highspeed steel HS 6-5-2-5 is shown in Fig. 4. It can be seen that the dominant frequencies in the two spectra generally coin- cide. This confirms the connection between the fluctuations of the cutting force and the temperature variations.

The theoretical and observed amplitudes of temperature variations were also compared. As a result of some calculation tests it was established that the temperature variations are proportional to the amplitude of the vibrations of the cutting force $F_{t}$ and are inversely proportional to the square root of the fluctuations frequency:

$$
A_{\Theta}\left(A_{F t}, v_{F t}\right)=k_{\Theta} \cdot \frac{A_{F t}}{\sqrt{v_{F t}}}
$$

where:

$\mathrm{k}_{\Theta}$ is the calculated coefficient $\left(k_{\odot}=51\right.$ for a pair of highspeed steel HS 6-5-2-5-CBN; $k_{\Theta}=61.3$ for a pair of titanium alloy Ti-6Al-4V-diamond, $\mathrm{k}_{\Theta}=54.4$ for pair steel $\mathrm{C} 45$ - electrocorundum white).

A comparison of the theoretical and experimental amplitudes of temperature variations $A_{\ominus}$ at dominant frequencies shows that the error of the mathematical model (12) does not exceed $5 \%$. Thus, by the magnitude of the cutting a)

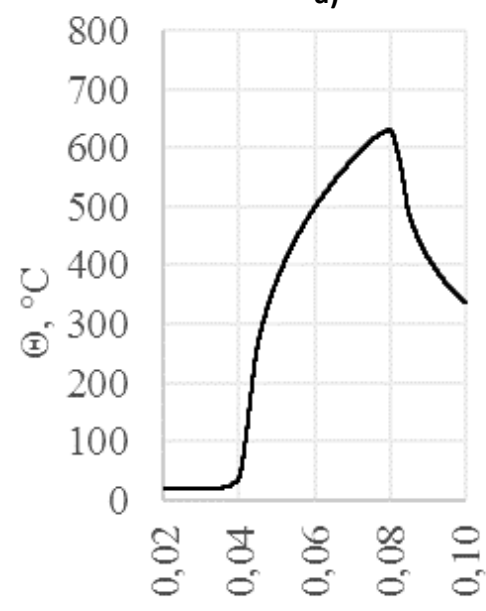

$\tau, \mathrm{S}$ b)

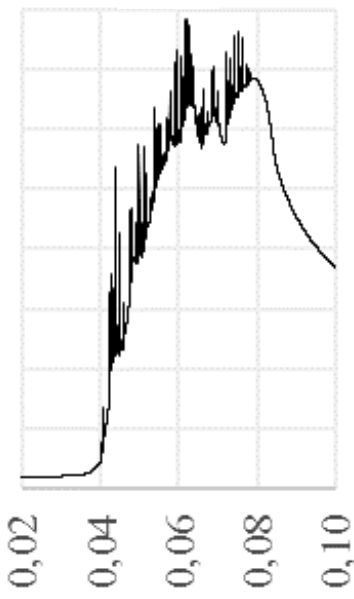

$\tau, \mathrm{S}$ c)

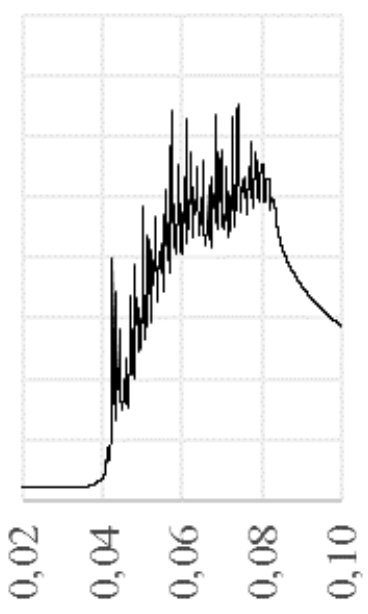

$\tau, \mathrm{s}$

Fig. 3 Theoretical curves of the grinding temperature variations
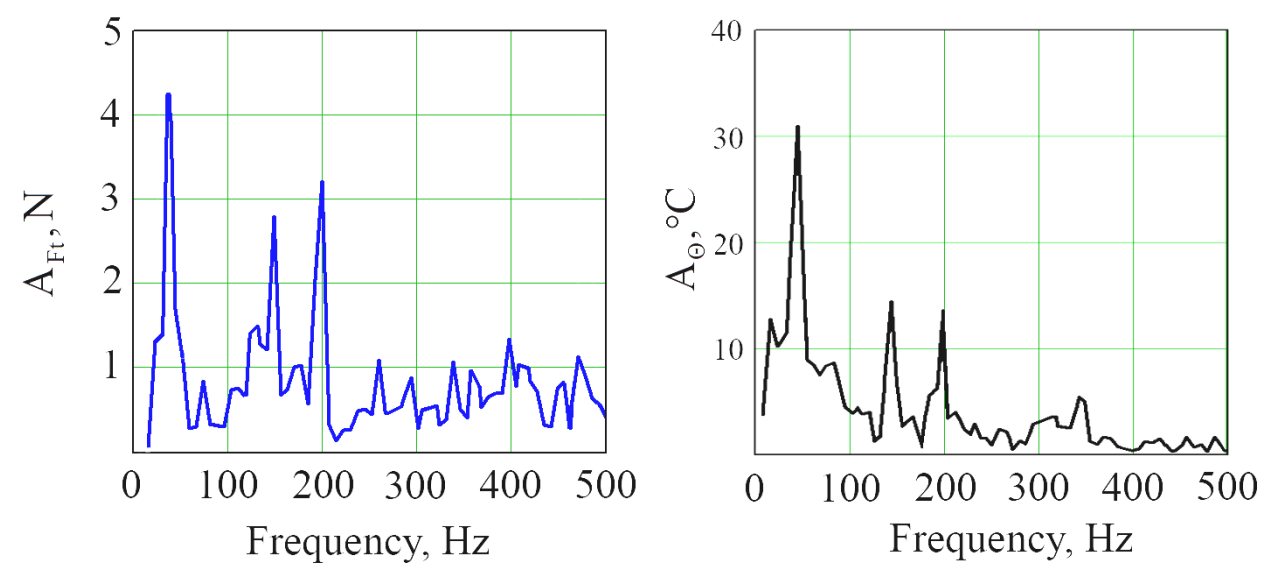

Fig. 4 The vibration spectra of $F_{t}$ and $\theta$

(workpiece material - steel HS 6-5-2-5, grinding wheel - electrocorundum white, ceramic bond, grit - F54; $R=100 \mathrm{~mm} ; V_{f}=2.5 \mathrm{~m} / \mathrm{min}$, $a_{p}=0.02 \mathrm{~mm}, V=35 \mathrm{~m} / \mathrm{s}$ ) 


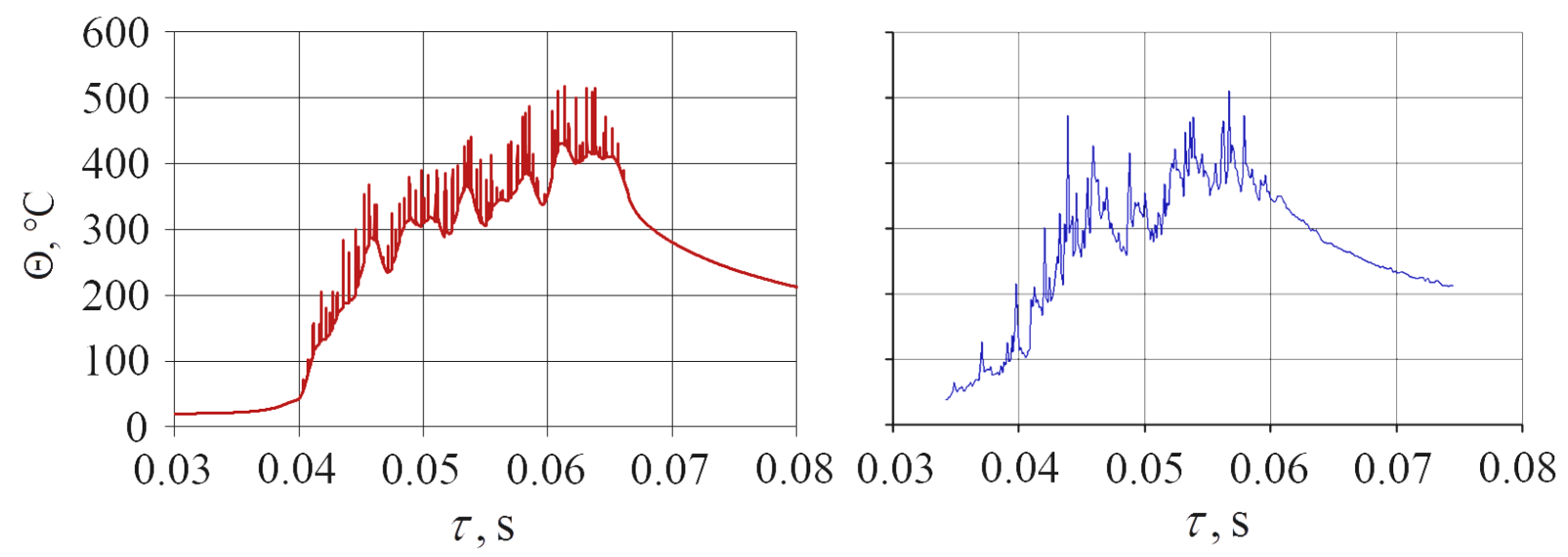

Fig. 5 Theoretical and experimental graphs of the grinding temperature

(workpiece material - tool steel 107WCR5, grinding wheel - electrocorundum white, ceramic bond, grit - F54, V = $35 \mathrm{~m} / \mathrm{s}$, $V f=3 \mathrm{~m} / \mathrm{min}, a p=0.01 \mathrm{~mm}$ )

force fluctuations, it is possible to predict the variations in the grinding temperature.

b. Comparison of the observed signal and the theoretical graph of the grinding temperature variations.

Based on the results of measuring the magnitude and the cutting force fluctuations, the input characteristics for grinding temperature calculating were determined. Next, the most suitable value of the coefficient $k_{u}$ (formula (7)) was chosen, at which the theoretical and experimental results have the best matching. Fig. 5 shows an example of a comparison of the theoretical (left) and experimental graphs (right) of the grinding temperature of tool steel 107 WCR5.

The graphs clearly show low-frequency temperature variations from the action of variable cutting force and high -frequency temperature variations from single grains cutting.

These researches were carried out for various workpiece materials. It was found that the discrepancy between the theoretical and experimental values of the grinding temperature does not exceed $5 \%$. Thus, the proposed thermophysical model and its computer implementation make it possible to simulate quite reliably the thermal processes occurring in the cutting zone.

\section{CONCLUSIONS}

The developed thermophysical model of flat peripheral grinding and the software can be used for the following.

1. Selection and optimization of grinding modes and the grinding wheel parameters when processing a given material.

2. Select the parameters of special grinding wheels (intermittent, perforated) for processing the given material.

3. Evaluation of the influence of vibrations in the technological system on the grinding temperature variations. Determination of the moment for dressing the grinding wheel by the signal of vibroaccelerometers.

4. Evaluation of the peak grinding temperature, depending on the parameters of the grinding wheel and cutting modes.

\section{ACKNOWLEDGEMENTS}

This research was supported by a grant from the state non-commercial organization

"The Foundation for Assistance to Small Innovative

Enterprises in the Scientific and Technical Sphere» (Russian Federation).

\section{REFERENCES}

[1] Z. Ding, B. Li and S.Y. Liang. "Phase transformations and residual stress of Maraging C250 steel during grinding", Materials Letters, vol. 154, pp. 37-39, Sep. 2015.

[2] V.F. Formalev and D.L. Reviznikov. Numerical methods. Moscow: FIZMATLIT, 2004.

[3] R.J. Gu, M. Shillor, G.C. Barber and T. Jen. "Thermal analysis of the grinding process", Mathematical and Computer Modelling, vol. 39, pp. 991-1003, May 2004.

[4] J. Kulka, M. Mantic, G. Fedorko and V. Molnár. "Analysis of crane track degradation due to operation", Engineering Failure Analysis, vol. 59, pp. 384395, Jan. 2016.

[5] A. Lefebvre, P. Lipinski, P. Vieville and C. Lescalier. "Experimental analysis of temperature in grinding at the global and local scales", Machining Science and Technology, vol. 12, no. 1, pp. 1-14, Mar. 2008.

[6] S. Malkin and C. Guo. "Thermal Analysis of Grinding", CIRP Annals, vol. 56, no. 2, pp. 760-782, Nov. 2007.

[7] I.D. Marinescu, M. Hitchiner, E. Uhlmann, W.B. Rowe and I. Inasaki. Handbook of machining with grinding wheels. Boca Raton, FL: CRC Press Taylor \& Francis Group, 2006.

[8] V.A. Nosenko and S.V. Nosenko. Metal grinding technology. Stariy Oskol: TNT, 2016.

[9] E. Pivarčiová and P. Božek. "Registration of holographic images based on integral transformation", Computing and informatics, vol. 31, no. 6, pp. 1369-1383, 2012.

[10] N. Ortega, H. Bravo, I. Pombo, J.A. Sanchez and G. Vidal. "Thermal analysis of creep feed grinding", Procedia Engineering, vol. 132, pp. 1061-1068, 2015.

[11] V.A. Smirnov. "The influence of geometric error of the grinding wheel and technological system vibrations on 
the chip thickness change by peripheral flat grinding", Bulletin of ISTU, vol. 3, pp. 16-18, Mar. 2008.

[12] V. Sviatskii, A. Repko, D. Janačova, Z. Ivandič, O. Perminova and Y. Nikitin. "Regeneration of a fibrous sorbent based on a centrifugal process for environmental geology of oil and groundwater degradation", Acta Montanistica Slovaca, vol. 21, no. 4, pp. 272-279, 2016.

[13] R. Grega, J. Krajnak, L. Zulova, G. Fedorko and V. Molnár. "Failure analysis of driveshaft of truck body caused by vibrations", Engineering Failure Analysis, vol. 59, pp. 208-215, Sept. 2017.

[14] J. Černecký, K. Valentová, E. Pivarčiová and P. Božek. "Ionization Impact on the Air Cleaning Efficiency in the Interior", Measurement Science Review, vol. 15, no. 4, pp. 156-166, 2015.

\footnotetext{
Assoc. prof. Vitalii A. Smirnov Ph.D., Prof. Aleksandr V. Repko. D.Sc.

Votkinsk Branch of Kalashnikov Izhevsk State Technical University

Department Technology of Mechanical Engineering and Instrument Making

Studencheskaya 7, Izhevsk 426069, RUSSIA

e-mail: smivial@mail.ru

aleksrepko@gmail.com
} 